\title{
Predicting efficacy of sunitinib in metastatic renal cell carcinoma
}

This article was published in the following Dove Press journal:

Current Biomarker Findings

29 May 2014

Number of times this article has been viewed

\author{
Carlo Ganini, ${ }^{1, *}$ \\ Camillo Porta ${ }^{1,2, *}$ \\ 'Medical Oncology, Istituto di \\ Ricovero e Cura a Carattere \\ Scientifico (IRCCS) San Matteo \\ University Hospital Foundation, \\ 'Italian Group of Nephro-Oncology/ \\ Gruppo Italiano di Oncologia \\ Nefrologica, Pavia, Italy \\ *Both authors contributed equally to \\ this paper
}

Correspondence: Camillo Porta Medical Oncology, IRCCS, San Matteo University Hospital Foundation, Piazzale C Golgi I9, I-27I00 Pavia, Italy

Tel $+39038250 \quad$ I 355

$\mathrm{Fax}+390382525240$

Email c.porta@smatteo.pv.it

\begin{abstract}
Renal cell carcinoma accounts for 3\% of all solid neoplasms in adults. Once poorly treated due to its poorly understood pathogenesis, the discovery of the role played by the vascular endothelial growth factor pathway in renal cell carcinoma has led to development of a number of targeted therapies that have impacted on the natural history of the disease. One of the problems related to this apparent abundance of therapies is choice of a drug tailored to the individual patient. Of all the drugs available, sunitinib accounts for more than $50 \%$ of first-line therapy. Defining which group of patients will benefit from sunitinib using a predictive biomarker would be of great help for its clinical activity. Local efforts have identified biomarkers that are potentially predictive of the efficacy of sunitinib in patients with metastatic renal cancer, being either clinical (hypertension), cellular (such as circulating endothelial cells, circulating tumoral cells), or molecular (cytokines such as vascular endothelial growth factor, hepatocyte growth factor/scatter factor), but there is a desperate need to increase the numbers of patients in the studies being conducted to provide more valid and reproducible data of use in clinical decision-making regarding therapy.
\end{abstract}

Keywords: kidney cancer, sunitinib, predictive biomarker, target therapy, tyrosine kinase inhibitor

\section{Introduction}

Renal cell carcinoma (RCC) accounts for approximately 3\% of all solid neoplasms in adults and represents the seventh leading cause of death from cancer worldwide, with the clear-cell histotype representing about $80 \%$ of all cancers arising from the kidney. ${ }^{1}$ In 2006, more than 209,000 new cases of RCC and 102,000 related deaths occurred worldwide; in Europe, the incidence of RCC has been estimated to be 29,600 new cases per year in men and 16,700 new cases per year in women. ${ }^{2}$

A better understanding of the molecular pathogenesis of this cancer has been the first essential step towards development of active therapeutic strategies, even though a lot remains to be clarified regarding the complex mechanisms leading to development of RCC. ${ }^{3}$ Some risk factors have been identified on the basis of meta-analysis of case-control studies and cohort studies showing a strong association with tobacco use and obesity. ${ }^{4}$ Further, characterization of the molecular pathogenesis of this cancer has highlighted the occurrence of crucial events, such as germline mutation of the oncosuppressor von Hippel-Lindau (VHL) gene, encoded on chromosome 3, ${ }^{5,6}$ which is associated with an increased incidence of RCC in patients with VHL syndrome, that interferes with the hypoxia-inducible factor (HIF)- $1 \alpha$ pathway ${ }^{7,8}$ and ultimately leads to overproduction of proangiogenic growth factors, such as vascular endothelial 
growth factor (VEGF), with the development of a new vessel network that feeds tumor cells.

Notably, there is a discrepancy in the slope of the curves for mortality and incidence of this cancer, partly due to the improvement in treatment that has occurred in the last decade, and of course also to an increased number of potentially curable small masses diagnosed by chance as a result of widespread use of noninvasive radiological techniques, eg, ultrasonography. Despite this, there has been a continuous, slow, but evident increase in the incidence of RCC, estimated at $2 \%-3 \%$ per decade, with a cumulative increase in incidence of $126 \%$ from 1950 until the present time. ${ }^{9}$

Therapeutic improvements have translated into an improved overall survival of $64 \%$ at 5 years in 2002 versus $40 \%$ in the early $1960 \mathrm{~s},{ }^{10}$ and the recent availability of novel agents targeting the molecular hallmarks of RCC will probably improve these figures further.

Intensive research has focused on prognostic factors able to predict the outcome of the disease independent of treatment, but the most effective thus far is still the stage of disease at initial diagnosis. Five-year overall survival is $80 \%-100 \%$ in patients with localized disease, but is only $12 \%$ in patients with distant metastasis, and $20 \%-25 \%$ of this population shows one or more metastatic sites at initial diagnosis. ${ }^{2}$

A lot has changed in the last decade from a therapeutic viewpoint, with metastatic RCC having moved from an orphan disease whereby cytokine therapy benefited only a minority of patients ${ }^{11}$ to a cancer now amenable to a number of treatment options. ${ }^{12} \mathrm{RCC}$ is indeed considered a pioneering field for the development of targeted therapies, with all current rational and accepted therapies used in the metastatic setting being tyrosine kinase inhibitors (TKIs) or monoclonal antibodies, either directed towards the signaling pathway mediated by VEGF and its receptor (VEGFR) or the mammalian target of rapamycin (mTOR) pathway. ${ }^{13}$ Experimental development of new drugs directed towards these two principal pathways has led to the possibility of treating patients with $\mathrm{RCC}$ using at least six active drugs, ie, bevacizumab plus interferon- $\alpha,{ }^{14}$ sorafenib, ${ }^{15}$ sunitinib, ${ }^{16}$ pazopanib,${ }^{17}$ axitinib,${ }^{18}$ temsirolimus,${ }^{19}$ and everolimus. ${ }^{13}$ Although local regulatory bodies can provide some guidance on use of these compounds, choosing first-line treatment is always a challenge for clinicians, ${ }^{20}$ who are required to choose between drugs that have rarely been compared with each other in randomized clinical trials, and thus base their decision on clinical experience alone.

Molecular targeting agents bring as a corollary that, if the target is so well defined from a biologic viewpoint (a receptor rather than an intracellular molecule for signal transduction), the patient himself should be "targeted", selected on the basis of biomarkers of any nature, that could help to define an appropriate patient population that will benefit from one drug instead of another. Scientific research should now be directed towards defining clinical strategies to select the appropriate drug for the individual patient and avoid exposure of patients with RCC to drugs that will not produce any objective effect (or indeed cause harm) according to the history of their disease, and avoid the use of drugs that are ineffective, with a high cost to be sustained, either by the local health system or by the subjects himself.

Clinical research has focused on evaluation of prognostic models that are able to predict the outcome for a patient independent of treatment, leading initially to use of the Memorial Sloane Kettering Cancer Center score (developed during the cytokine era) ${ }^{21,22}$ to predict the outcome in patients with metastatic disease. This scoring system was subsequently revised by Heng et al in $2009^{23}$ and validated in a population of patients treated with molecularly targeted agents outside clinical trials. However, there has been insufficient effort made in trying to predict the efficacy of a given therapy in a given patient.

The agent most frequently used in first-line treatment for RCC continues to be sunitinib, a multitargeted TKI, the efficacy of which has been established by a randomized controlled trial versus interferon- $\alpha$, which was the standard of care in the pretargeted therapy era. ${ }^{16,24,25}$

The lack of predictive biomarkers available for a priori selection of patients likely to derive clinical benefit from sunitinib, ie, disease stabilization or an objective response, has led to an intensive search for prognostic biomarkers. ${ }^{26}$ The aim of this review is to present and revise the studies conducted, giving a systematic insight into the present status of the search for predictive biomarkers in RCC patients treated with sunitinib.

\section{Methods}

Studies of interest were identified via a database search of PubMed and the Cochrane Library, also searching for relevant abstracts in annual conference proceedings up to September 2013. The search terms used were "sunitinib", "biomarker", "kidney", "predictive", and "prognosis", combined with "carcinoma", "cancer", and "tumor" using the Boolean operator "AND". In addition, we manually searched the papers of interest to identify those most pertinent to the aim of our review. 


\section{Clinical biomarkers Hypertension}

Hypertension is one of the most frequently observed toxicities in patients treated with sunitinib, ${ }^{27}$ and indeed with almost all anti-VEGR/VEGFR TKIs, and has been proposed as a predictive biomarker of drug activity. Inhibition of VEGF is likely to cause hypertension via interruption of endothelial cell survival signaling and subsequent induction of apoptosis, associated with decreased capillary number, and via inhibition of production of nitric oxide by endothelial cells, leading to constriction of the vascular smooth muscle cells of blood vessels and activation of the endothelin-1 pathway. ${ }^{28}$ In a retrospective analysis of 544 sunitinib-treated patients with metastatic RCC, $81 \%$ and $67 \%$ experienced systolic or diastolic hypertension, respectively; patients who developed hypertension (defined as a systolic blood pressure $>140 \mathrm{mmHg}$ or a diastolic blood pressure $>90 \mathrm{mmHg}$ ) had an improved outcome, with an odds ratio of $54.8 \%$ in patients with hypertension versus $8.7 \%$ in those without hypertension $(P<0.001)$. Median progression-free survival was 12.5 months ( $95 \%$ confidence interval [CI] 10.9-13.7) for hypertensive patients versus 2.5 months (95\% CI 2.3-3.8) for patients without significant blood pressure changes; similarly, median overall survival was 30.9 months (95\% CI 27.9-33.7) versus 7.2 months (95\% CI 5.6-10.7), respectively. Multivariate analysis demonstrated that hypertension is a viable and independent biomarker of the antitumor efficacy of sunitinib in patients with RCC..$^{29}$ The major critical point regarding the role of hypertension as a prognostic marker remains the accuracy of blood pressure monitoring, which is potentially prone to measurement errors depending on how, where, and by whom it is measured.

\section{Hypothyroidism}

Remaining in the field of sunitinib-related adverse events as possible predictive biomarkers for response to therapy, hypothyroidism, which is a well known effect linked to sunitinib therapy, ${ }^{30}$ has been considered in many studies. ${ }^{31}$ The way in which sunitinib induces hypothyroidism is not well understood; it possibly causes an increase in apoptosis of follicular cells with subsequent destructive thyroiditis; this action, together with endothelial dysfunction, inhibits iodine uptake by the thyroid gland, leading to reduced synthesis of thyroid hormones. ${ }^{31}$

The first attempt comes from the work of Wolter et al, which showed a possible correlation of outcome with the development of hypothyroidism in 40 patients with metastatic RCC treated with sunitinib. Seventy percent of the patients developed hypothyroidism; median progressionfree survival was 3.6 months (95\% CI 2.3-6.0) in patients who did not develop hypothyroidism versus 10.3 months (95\% CI 5.0-18.0) in presence of thyroid abnormalities $(P=0.047)$, and overall survival was 6.6 months $(95 \% \mathrm{CI}$ $3.3-7.9)$ in the former group versus 18.2 months $(95 \% \mathrm{CI}$ 7.5-22.3) in the latter group $(P=0.13))^{32}$ However, hypothyroidism as a predictive tool is controversial, as shown by Sabatier et al in a study conducted in 111 patients with metastatic RCC treated with sunitinib. In their study, hypothyroidism was confirmed as a common adverse event of sunitinib, but was not correlated with progression-free survival (18.9 months versus 15.9 months in patients who developed hypothyroidism and those who did not, respectively; hazard ratio [HR] $1.02,95 \% \mathrm{CI} 0.54-1.93 ; P=0.94){ }^{33}$

\section{Hand-foot syndrome}

Hand-foot syndrome is a common adverse event of antiVEGF treatment, particularly with sorafenib but also with sunitinib (occurring in up to $9 \%$ of the treated population). The pathogenesis is not understood, but some have suggested a role played by death of endothelial cells in the dermis as well as inhibition of KIT, a molecule present at high density in the eccrine glands of the sites most frequently involved in the syndrome. ${ }^{31}$

In a large retrospective study, Puzanov et al reviewed 770 cases of metastatic RCC (as well as 416 cases of gastrointestinal stromal tumor) treated with sunitinib, and reported that the group with metastatic RCC and development of handfoot syndrome had better clinical outcomes, with a longer overall survival (38.2 months in the hand-foot syndrome group versus 18.9 months in the group without; $P<0.001$ ) as well as prolonged progression-free survival (14.3 months versus 8.3 months, respectively; $P<0.001) .{ }^{34}$ The data remained valid after multivariate analysis, showing that hand-foot syndrome is a significant predictor of progression-free survival (HR $0.75,95 \%$ CI $0.60-0.94 ; P=0.0148$ ) and overall survival (HR 0.58, 95\% CI 0.44-0.77; $P<0.0001$ ). ${ }^{35}$

Hand-foot syndrome should be regarded with caution as a biomarker, not only because it is an unpredictable adverse event, but also because it can be effectively prevented from the start of treatment with anti-VEGF therapies, eventually interfering with its development.

\section{Biological biomarkers Cells as biomarkers}

Many cell populations are potentially involved in the pathogenesis of RCC, including those of the immune system, ${ }^{36}$ 
as well as fibroblasts, endothelial cells, circulating tumor cells, and hematopoietic progenitor cells. Among all of the cell populations investigated, circulating endothelial cells ${ }^{37}$ show possible interactions with the tumor environment, establishing a niche for the development of the tumor. ${ }^{38}$ Circulating endothelial cells have been considered as possible biomarkers, and it is thought they may be the result of shedding from mature endothelial cells after injury by anti-VEGF treatment.

\section{Circulating endothelial and tumoral cells}

Rossi et al ${ }^{39}$ studied circulating cells isolated from peripheral blood of patients who received sunitinib as first-line therapy in the metastatic setting and showed that changes in circulating tumoral cells mirrored changes in circulating endothelial cells measured during treatment with sunitinib; a rapid increase of the circulating endothelial cells together with a decrease in circulating tumoral cells has been linked to better outcome, opposed to a delayed increase of circulating endothelial cells, together with higher circulating tumoral cell values in the group with worst prognosis. This work also shows that not only the number of circulating cells, but also their viability (established by an apoptosis assay) is correlated with clinical outcome in patients treated with sunitinib, ie, those who have high levels of circulating tumoral cells, in high percentage apoptotic, had a better outcome in comparison with patients who had viable circulating tumoral cells. ${ }^{39}$

Treatment with sunitinib is associated with an increase in levels of circulating endothelial cells as an early biological response, as was seen in a study of 26 patients with metastatic RCC reported by Gruenwald et al. ${ }^{40}$ In their study, treatment with sunitinib increased circulating endothelial cell levels after only 14 days $(P=0.0331)$ and even more so after 28 days $(P=0.0159)$. A trend in favor of a correlation between such an increase (interpreted as an indirect sign of vascular damage, ie, an optimal result of anti-VEGF/VEGFR treatment) and progression-free survival was observed, even though statistical significance was not reached $(P=0.4414)$.

\section{Hematopoietic progenitor cells}

Hematopoietic progenitor cells are an interesting cell population to study because it has been shown that they are present at higher levels in patients with metastatic RCC. The mechanism by which this increase occurs is not known, but it has been suggested that this cell population moves from the bone marrow because it is stimulated by molecules secreted by tumoral cells, such as insulin-like growth factor- $1 .{ }^{38}$

In a study reported by Powles et al, 43 patients with metastatic RCC assessed as having an intermediate or poor prognosis according to Memorial Sloane Kettering Cancer Center score were tested for hematopoietic progenitor cells circulating in peripheral blood. Univariate and multivariate analyses showed that high levels of hematopoietic progenitor cells before treatment were associated with a significantly shorter overall survival (HR 3.3, 95\% CI 1.23-8.8; $P=0.01)$. These levels decreased by $61 \%$ during treatment with sunitinib $(P<0.05)$ and were correlated with prognosis, with subjects showing higher levels of hematopoietic progenitor cells having the worst prognosis. ${ }^{41}$ Given that this study, someway surprisingly, did not include patients with a good prognosis, it is impossible to extend this observation to the general metastatic RCC population, thus missing one important, even if not numerous, subgroup. The study also fails to distinguish between the prognostic or predictive value of hematopoietic progenitor cell levels in patients treated with sunitinib, but the fall in hematopoietic progenitor cells seen with sunitinib seemed not to correlate with the outcome. Further investigations are needed to evaluate this hypothesis.

\section{Molecular biomarkers}

Passing from cellular to molecular biology, many molecules have been studied to predict response to sunitinib, including cytokines or growth factors and genetic alterations, such as mutations in the genes encoding for proteins in the VHL/ HIF- $1 \alpha$ axis observed in RCC, as well as epigenetic modulation of gene expression.

\section{Genes}

Very few attempts have been made to identify any correlation between genetic alterations and the outcome in patients receiving targeted anti-VEGF therapy. Choueiri et al have published some relevant data on mutations of the VHL gene in tissue specimens from primary kidney cancer. Their study population consisted of 123 subjects treated with anti-VEGF/VEGFR therapies (not only sunitinib) who showed a response rate of $37 \%$ (95\% CI 28-46), a median progression-free survival of 10.8 months (95\% CI 7.7-14.8), and a median overall survival of 29.8 months. Patients with inactivation of VHL had a response rate of $41 \%$ versus $31 \%$ for patients with wild-type VHL $(P=0.34)$. Loss of function mutations were also evaluated, showing a $52 \%$ response 
rate in the loss of function group versus a 31\% response rate for the wild-type VHL group $(P=0.04) .{ }^{42}$ Progression-free survival and overall survival were not significantly different between the two groups, but further investigation of VHL is needed.

\section{Molecular or epigenetic changes in VEGF or related receptors}

It is important to focus on the genome as well as on the epigenetic changes that could markedly modify gene expression. A preclinical study by Kim et al focused on methylation of the promoter regions of the VEGFR genes (Flt1 and KDR), and greater inhibition of proliferation by TKIs (in their study represented by PTK/ZK and sunitinib) was observed in some cancer cell lines, which showed no promoter methylation for either promoter gene. The methylation status of these genes has been shown to favor the activity of intracellular agents such as sunitinib in the presence of a nonmethylated promoter, underscoring the role played by epigenetic changes, confirmed by demethylation experiments, in which the methylation levels of the promoters were artificially diminished before treatment with a TKI, leading to increased inhibition of proliferation by TKIs in cell lines that did not show such behavior when hypermethylated. ${ }^{43}$

Sunitinib is approved for use after failure or intolerance of imatinib in gastrointestinal stromal tumors. A study conducted by Heinrich et al in this population showed that another kinase inhibited by sunitinib, ie, KIT, or more specifically the gene that encodes for it, behaves as a biomarker of efficacy for sunitinib. The population was studied for both primary mutations of the KIT gene (in a specimen from the primary tumor before treatment with imatinib) and secondary mutations (after failure of imatinib but before starting sunitinib), and showed a clinical benefit from treatment with sunitinib (defined either by partial response or by stable disease for more than 6 months) in the groups of patients with three common primary genotypes (KIT exon 9 mutation, 58\%; KIT exon 11 mutation, 34\%; and wild-type KIT, $56 \%$ ), with a longer progression-free survival and overall survival for patients with primary KIT exon 9 mutation $(P<0.0005)$ or with a wild-type genotype $(P<0.0356)$. Considering mutations that occurred during treatment with imatinib, KIT exon 13 or 14 mutations showed clinical benefit rather than exon 17 or 18 mutation. ${ }^{44}$ Although not directly including a population with metastatic RCC, this study is extremely important because it sheds light on the role played by epigenetics, and it would be reasonable to collect the same data in a pure metastatic RCC population in the future.

\section{Cytokines and their receptors}

In a pioneer study published by Deprimo et al, plasma levels of four soluble proteins, ie, VEGF, soluble VEGFR-2, placental growth factor, and soluble VEGFR-3 (proteins involved in angiogenesis), ${ }^{45}$ were measured in a cohort of 63 patients with metastatic RCC treated in a Phase II study of sunitinib in a post-cytokine setting, with samples taken at fixed intervals during the first four cycles of therapy.

VEGF and placental growth factor were chosen, among other soluble molecules, because they are actively secreted during the process of angiogenesis, possibly in response to activation of HIF-1 $\alpha$, as sunitinib activity leads to interference with angiogenesis. Placental growth factor, which is not directly inhibited by sunitinib, (as opposed to the VEGF pathway), shows correlations with the outcome of the treatment. Compared with baseline, VEGF and placental growth factor levels increased by more than three-fold in $44 \%$ and $40 \%$ of patients, respectively $(P<0.001)$. Soluble VEGFR-2 levels decreased by more than $30 \%$ in $91 \%$ of the population and by more than $20 \%$ in all other cases $(P<0.001)$ during the first cycle of therapy, while soluble VEGFR-3 levels decreased by more than $30 \%$ in $87 \%$ of cases, and by more than $20 \%$ in all but two patients. These levels tended to return almost to baseline after the typical pause of 2 weeks off, indicating that these effects probably depend on drug exposure. Overall, major changes in VEGF, serum soluble VEGFR-2, and soluble VEGFR-3 levels were noticed in patients who showed an objective tumor response rather than in those with stable or progressive disease ( $P<0.05$ for each protein considered). These changes have been described as likely being directly related to the activity of sunitinib and, at least in part, to inhibition of the VEGF pathway. ${ }^{46}$

VEGF alone is a promising tool for predicting the outcome for patients treated with sunitinib, but it has also been considered along with other molecules, not necessarily cytokines, such as neutrophil gelatinase-associated lipocalin (NGAL), ${ }^{47}$ a protein typically upregulated in cells disturbed by a stressed environment, such as in the presence of a tumor. NGAL is not directly connected with the VEGF pathway, but its role in stressed environments identifies it as a possible candidate for predicting the efficacy of an antitumor drug such as sunitinib. Our group measured VEGF and NGAL levels in peripheral blood at predetermined intervals in a population of 85 patients with metastatic RCC who were treated with sunitinib, and found that their baseline levels were significantly 
predictive of progression-free survival, with a relative risk of 1.0004 and 1.004, respectively, for a unitary increase of each parameter. Patients were then divided into two groups according to whether their VEGF and NGAL values were above or below the threshold (chosen as the upper limit of the normal range). The relative risk of progression was 2.14 (95\% CI 1.324-3.459) in the group of patients with a VEGF value below the threshold. NGAL also showed a separation line close to the upper value of normal, with a relative risk of progression of $1.86(95 \%$ CI $1.142-3.019)$ relative to patients with values below this threshold. Further, patients with VEGF above the threshold had a median progression-free survival of 4.7 months (95\% CI 2.8-8.3), whereas patients with VEGF below the threshold had a median progression-free survival of 11.2 months (95\% CI 6.5-15). Patients with NGAL above the threshold had a median progression-free survival of 3.35 months (95\% CI 2.3-10.9), whereas those with NGAL below the threshold had a median progression-free survival of 8.15 months (95\% CI 5.5-11.6). ${ }^{48}$

These data suggest the possibility of integration of biomarkers in a strategy that can create a "system of biomarkers", rather than trying to define a single one; such a biomarker system would be more difficult to use but could reflect the complexity of the pathogenesis of this disease and, moreover, the complexity of the molecular mechanisms elicited by target therapies themselves, eventually leading to evolution of resistance to a given therapy, such as sunitinib. ${ }^{49}$

Interestingly, other cytokines considered to be involved in the mechanisms of resistance to sunitinib, including basic fibroblast growth factor, ${ }^{50}$ interleukin- $6,{ }^{51}$ and hepatocyte growth factor/scatter factor, ${ }^{52}$ have been studied as predictors of response to sunitinib because they have been shown to be possible molecular escape pathways after treatment with antiangiogenic drugs. Indeed, we showed in a study of patients with metastatic RCC treated using sunitinib that the above cytokines (basic fibroblast growth factor in particular) increased from baseline values immediately before radiological progression. ${ }^{53}$ This study had a merely descriptive aim, due to multiple possible confounding elements, including a limited sample size, that could have led to some false-positive results or the selection of only three cytokines thought to play a role in the development of resistance to sunitinib, among all the possibilities.

One other comprehensive way of studying cytokines to identify a biomarker potentially predictive of the efficacy of sunitinib, is the one described by Perez-Garcia et al who used microarray analysis for 174 cytokines simultaneously, initially stating which ones showed differences between the group of responders versus the group of nonresponders to sunitinib therapy; among the 174 cytokines, matrix metalloproteinase (MMP)-9, and tumor necrosis factor (TNF)- $\alpha$ showed significant differences between the two populations. This lead to the decision of dosing them among all 21 patients included in the study. MMP-9 is activated by VEGF and SDF-1 and releases soluble KIT ligand, allowing the mobilization of endothelial and hematopoietic stem cells. It is associated with invasiveness and motility in a vast number of malignancies, possibly through its ability to degrade the extracellular matrix. Baseline levels of the cytokines, quantified by enzyme-linked immunosorbent assay, were compared with those measured at the time of radiological evaluation of the therapy. MMP-9 significantly changed during treatment whereas TNF- $\alpha$ did not. Sunitinib significantly decreased MMP-9 levels in both patient groups $(P<0.011$ for patients with clinical benefit and $P<0.005$ for patients with progression). ${ }^{54}$

\section{Soluble proteins other than cytokines}

Not only cytokines have been studied in an attempt to predict the efficacy of sunitinib in patients with metastatic RCC; other soluble proteins detected in peripheral blood have had their own attention in this field, including C-reactive protein, brain natriuretic peptide, and follicle-stimulating hormone.

The link between cancer and inflammation is a very important topic in cancer research today. This link has been recognized for decades, but is still not fully understood due to its complexity. Fujita et al used a marker of inflammation to evaluate the response to sunitinib, and evaluated C-reactive protein levels in 41 patients with metastatic RCC treated with sunitinib. C-reactive protein was evaluated not only for its correlation with objective response, but also for correlation with other clinical characteristics, such as Memorial Sloane Kettering Cancer Center score and some of the adverse events related to the drug. C-reactive protein was independently associated with objective response $(P=0.016)$. Patients with a normal level of C-reactive protein $(<0.30 \mathrm{mg} / \mathrm{dL})$ had significantly higher rates of partial response or disease stabilization ( $84.6 \%$ versus $35.7 \% ; P=0.002$ ), and had significantly longer progression-free survival (19.0 versus 6.0 months, $P=0.036)$ than patients with elevated $\mathrm{C}$-reactive protein, 
leading to the conclusion that $\mathrm{C}$-reactive protein is an independent prognostic biomarker in patients with metastatic RCC treated with sunitinib. ${ }^{55}$

Among the so-called surrogate biomarkers, plasma levels of N-terminal precursor of brain natriuretic peptide (NT-pro-BNP) were evaluated in 36 patients with metastatic RCC treated with sunitinib; even if NT-pro-BNP is not a molecule that interacts directly in angiogenesis as far as we know, its response to cardiac injury is interesting considering that we are talking about drugs, such as sunitinib, which have been shown to have toxic cardiac effects. In this study, nine of the 36 patients had progressive disease and 27 had a response to sunitinib; plasma NT-pro-BNP levels correlated with outcome, with patients who progressed having higher levels of NT-pro-BNP, especially during the first 15 days of treatment (with a three-fold increase in the group of patients with progressive disease compared to stable NT-pro-BNP levels in the other group, $P<0.0001)$. Median progressionfree survival was 12.0 months in patients with a less than 1.5-fold increase and 3.9 months in those with more than a 1.5 -fold increase, ${ }^{56}$ indicating that this molecule could be a predictive tool in determining the efficacy of sunitinib in metastatic RCC.

Finally, hormonal status has been taken into account in trying to predict efficacy as for the testing of folliclestimulating hormone receptor on primary kidney cancer, studying 50 patients treated with sunitinib for metastatic RCC. Follicle-stimulating hormone receptor was selected due to the evidence of its overexpression in tumoral vasculature, even if there is not a clear link between the pathogenesis of most of the cancer types from which it has been recognized, as well as for RCC. ${ }^{57}$ In this study, the density of follicle-stimulating hormone receptor, stained onto tumor vessels from primary kidney cancer in patients with metastatic RCC, and progression-free survival was 0.50 ( $\mathrm{n}=43, P<0.0005)$. Subsequent measurement revealed that the patients could be divided into three groups (ie, progressed, stable, and improved) differentiated by the ratio between follicle-stimulating hormone receptorpositive vessel density and total vessel density: in the group of the responders to sunitinib, the proportion of follicle-stimulating hormone receptor-positive vessels was five-fold higher than in the stable group (56.8 \pm 5.4 versus $11.4 \pm 2.0$, respectively), and almost eight-fold higher than in the progressed group $\left(P=3 \times 10^{-9}\right.$ in the improved versus stable patients, and $P=5 \times 10^{-16}$ in the improved versus progressed patients). The difference between the stable and progressed groups was statistically significant $(P<0.02)$. These data shed light on the possibility of predicting the outcome of treatment with sunitinib, based on the percentage of follicle-stimulating hormone receptor-stained vessels in the primary tumor. ${ }^{58}$

\section{Discussion}

As far as we know, metastatic RCC is a disease for which great therapeutic improvement has been obtained in the last decade. The discovery of the key involvement of the VEGFR pathway, mediated by hypoxia-induced signals, has led to the development of a variety of compounds, mainly TKIs directed against VEGFR or against mTOR (molecule intercalated with the hypoxia-mediated and phosphatidylinositol 3 phosphate-AKT pathways), that have partially changed the natural history of this disease, which was once destined to be fatal in a few months. New compounds have provided the opportunity to prolong progression-free survival and overall survival as well as created a new problem to solve, ie, after a failed treatment, which is the best option? Much effort has been made in trying to produce an ideal algorithm to optimally treat patients with metastatic RCC. ${ }^{59-61}$ What has emerged is that there is no perfect algorithm suitable for all patients, and that choosing the appropriate drug for an individual patient is the only way of optimizing therapy. Among all the possible choices, first-line therapy plays a crucial role. First-line therapy is now an extremely chaotic field in which the clinician has many potential weapons, the efficacy of which is of course not guaranteed in any given patient before the start of the therapy. Despite the activity of sunitinib, some patients would not benefit from this treatment. Predicting the efficacy of sunitinib would avoid important consequences such as toxicity, and ineffectiveness in a select group of patients. This will allow the saving of money, as target therapies are high-cost.

The scientific world has attempted a search for biomarkers predictive of a response to sunitinib, starting with clinical parameters such as hypertension, or going deeper into cellular biology and molecular biology (Table 1). Apart from case-by-case observations, what has emerged is that all those efforts have been conducted in small, local regions: in some studies, indeed, statistical significance has not been obtained, largely because of the small number of the subjects studied. The lack of statistically significant results makes it difficult to judge if those promising biomarkers are just prognostic or effectively predictive of the 
Table I Summary of potential biomarkers for predicting response to sunitinib

\begin{tabular}{|c|c|c|c|c|c|}
\hline $\begin{array}{l}\text { Biomarker } \\
\text { category }\end{array}$ & $\begin{array}{l}\text { Subgroup } \\
\text { biomarker }\end{array}$ & Biomarker & Reference & $\begin{array}{l}\text { Patient } \\
\text { population (n) }\end{array}$ & $\begin{array}{l}\text { Good prognosis group } \\
\text { based on biomarker }\end{array}$ \\
\hline \multirow[t]{4}{*}{ Clinical } & Vital signs & Hypertension & Rini et $\mathrm{al}^{29}$ & 544 & Patients with hypertension \\
\hline & & Hypothyroidism & Wolter et $\mathrm{al}^{32}$ & 40 & Patients with hypothyroidism \\
\hline & & Hypothyroidism & Sabatier et $\mathrm{al}^{33}$ & III & No differences between groups \\
\hline & & HFS & Puzanov et $\mathrm{a}^{34}$ & 770 & HFS-positive pts \\
\hline \multirow[t]{3}{*}{ Biological } & Cellular & CECs and CTCs & Rossi et $\mathrm{al}^{39}$ & 53 & Patients negative for CEC and CTC \\
\hline & & CECs & Gruenwald et a $\left.\right|^{40}$ & 26 & Patients positive for CEC \\
\hline & & HPCs & Powles et $\mathrm{al}^{41}$ & 43 & HPC-negative patients \\
\hline \multirow[t]{3}{*}{ Biological } & Genetic & $V H L$ & Choueiri et $\mathrm{a}^{42}$ & 123 & Patients with $V H L$ inactivation/loss of function \\
\hline & & $V H L$ methylation & Kim et $\mathrm{a}^{43}$ & $\begin{array}{l}\text { Not tested } \\
\text { in patients }\end{array}$ & Patients with nonmethylated $\mathrm{VHL}$ \\
\hline & & KIT & Heinrich et al ${ }^{44}$ & 78 GIST & $\begin{array}{l}\text { KIT exon } 9 \text { mutation-positive and wild-type } \\
\text { KIT for primary mutations; KIT exon } 13 \text { or } \\
\text { I4 mutation for secondary mutation patients }\end{array}$ \\
\hline \multirow[t]{4}{*}{ Biological } & $\begin{array}{l}\text { Molecular (cytokines } \\
\text { and receptors) }\end{array}$ & $\begin{array}{l}\text { VEGF, sVEGFR-2, } \\
\text { sVEGFR-3, PIGF }\end{array}$ & Deprimo et al ${ }^{45}$ & 63 & $\begin{array}{l}\text { VEGF-negative, sVEGFR-2-negative, sVEGFR- } \\
\text { 3-negative patients }\end{array}$ \\
\hline & & VEGF, NGAL & Porta et $\mathrm{al}^{48}$ & 85 & VEGF-negative, NGAL-negative patients \\
\hline & & bFGF, IL-6, HGF/SF & Porta et $\mathrm{a}^{53}$ & 85 & bFGF-negative patients \\
\hline & & TNF- $\alpha$, MMP-9 & Perez-Garcia et al ${ }^{54}$ & 21 & Patients negative for TNF- $\alpha$ and MMP-9 \\
\hline \multirow[t]{3}{*}{ Biological } & $\begin{array}{l}\text { Molecular (other } \\
\text { soluble proteins) }\end{array}$ & CRP & Fujita et a ${ }^{55}$ & 41 & CRP-negative patients \\
\hline & & NT-pro-BNP & Papazisis et $\mathrm{a}^{56}$ & 36 & NT-pro-BNP-negative patients \\
\hline & & FSHR & Siraj et $a^{58}$ & 50 & FSRH-positive pts \\
\hline
\end{tabular}

Abbreviations: VHL, von Hippel-Lindau; CECs, circulating endothelial cells; CTCs, circulating tumoral cells; HPCs, hematopoietic progenitor cells; VEGF, vascular endothelial growth factor; sVEGFR-2, soluble vascular endothelial growth factor receptor 2; sVEGFR-3, soluble vascular endothelial growth factor receptor 23; PIGF, placental growth factor; NGAL, neutrophil gelatinase-associated lipocalin; bFGF, basic fibroblast growth factor; IL-6, interleukin 6; HGF/SF, hepatocyte growth factor/scatter factor; TNF- $\alpha$, tumor necrosis factor $\alpha$; MMP-9, matrix metallo-proteinase 9; CRP, C-reactive protein; NT-pro-BNP, N-terminal precursor of brain natriuretic peptide; FSHR, folliclestimulating hormone receptor; HFS, hand-foot syndrome; GIST, gastrointestinal stromal tumor.

efficacy of a given therapy (such as sunitinib). Adverse events used as a biomarker, more than others, show good correlation with outcome and prognosis but represent the most fragile category, being impossible to predict which patient is going to develop any of the cited adverse events. Predicting the emergence of a possible predictive biomarker would add complexity to the already complex scenario. It has also emerged that it could be useful to consider a panel of biomarkers for use in a combined way rather than a single one; this approach may be even more difficult to implement, and could reflect the great complexity of molecular, cellular, and clinical changes that occur during the history of this disease and approximate best the response to therapy.

\section{Conclusion}

RCC is one of the most fascinating fields in clinical oncology for its close relationship to its molecular pathogenesis, which has permitted to prove the efficacy of many molecularly target therapies, able, in selected cases, to prolong life for years, but still failing in a larger number of patients. The problem of choice of the correct drug for a given patient has now to be solved: choosing among the many compounds available cannot be solely based on the clinician's expertise, which is obviously fundamental, but should be driven by objective predictive tools. The scientific community now has to coordinate research so that larger numbers of patients treated under the same conditions and with the same drugs, sunitinib for instance, can be tested, producing data that will be reproducible and applicable to many situations and provide important evidence on prognosis from both a local perspective and a worldwide one, making it easier to select a drug or, on the counterpart, to not select it.

\section{Acknowledgment}

Many thanks are extended to the clinical trials office at our institution, in particular Alessandra Ferrari, Francesca Reversi, and Simona Morabito, who supported the writing of this paper and gave the authors time to think and write this paper, and provided technical support for clinical research.

\section{Disclosure}

$\mathrm{CG}$ has no conflicts of interest to disclose. CP has acted as a consultant and/or speaker for Pfizer Oncology, GSK, 
Bayer-Schering Pharma, Novartis Pharma, Astellas, Aveo, and Boehringer-Ingelheim, and has received research support from Bayer-Schering Pharma and Novartis Pharma.

\section{References}

1. Gore ME, Szczylik C, Porta C, et al. Safety and efficacy of sunitinib for metastatic renal-cell carcinoma: an expanded-access trial. Lancet Oncol. 2009;10(8):757-763.

2. Gupta K, Miller JD, Li JZ, Russell MW, Charbonneau C. Epidemiologic and socioeconomic burden of metastatic renal cell carcinoma (mRCC): a literature review. Cancer Treat Rev. 2008;34(3):193-205.

3. Cheng L, Zhang S, MacLennan GT, Lopez-Beltran A, Montironi R. Molecular and cytogenetic insights into the pathogenesis, classification, differential diagnosis, and prognosis of renal epithelial neoplasms. Hum Pathol. 2009;40(1):10-29.

4. Dhote R, Thiounn N, Debré B, Vidal-Trecan G. Risk factors for adult renal cell carcinoma. Urol Clin North Am. 2004;31(2):237-247.

5. Kovacs G, Brusa P, De Riese W. Tissue-specific expression of a constitutional 3;6 translocation: development of multiple bilateral renal-cell carcinomas. Int J Cancer. 1989;43(3):422-427.

6. Peruzzi B, Athauda G, Bottaro DP. The von Hippel-Lindau tumor suppressor gene product represses oncogenic beta-catenin signaling in renal carcinoma cells. Proc Natl Acad Sci U S A. 2006;103(39): 14531-14536.

7. Melillo G. Targeting hypoxia cell signaling for cancer therapy. Cancer Metastasis Rev. 2007;26(2):341-352.

8. Semenza GL. Hypoxia-inducible factor 1 and cancer pathogenesis. IUBMB Life. 2008;60(9):591-597.

9. Mathew A, Devesa SS, Fraumeni JF, Chow W-H. Global increases in kidney cancer incidence, 1973-1992. Eur J Cancer Prev. 2002;11(2): 171-178.

10. Clegg LX, Reichman ME, Miller BA, et al. Impact of socioeconomic status on cancer incidence and stage at diagnosis: selected findings from the surveillance, epidemiology, and end results: National Longitudinal Mortality Study. Cancer Causes Control. 2009;20(4):417-435.

11. [No authors listed]. Interferon-alpha and survival in metastatic renal carcinoma: early results of a randomised controlled trial. Medical Research Council Renal Cancer Collaborators. Lancet. 1999;353(9146):14-17.

12. Vogelzang NJ. Treatment options in metastatic renal carcinoma: an embarrassment of riches. J Clin Oncol. 2006;24(1):1-3.

13. Motzer RJ, Escudier B, Oudard S, et al. Efficacy of everolimus in advanced renal cell carcinoma: a double-blind, randomised, placebocontrolled phase III trial. Lancet. 2008;372(9637):449-456.

14. Yang JC, Haworth L, Sherry RM, et al. A randomized trial of bevacizumab, an anti-vascular endothelial growth factor antibody, for metastatic renal cancer. $N$ Engl J Med. 2003;349(5):427-434.

15. Escudier B, Eisen T, Stadler WM, et al. Sorafenib in advanced clear-cell renal-cell carcinoma. $N$ Engl J Med. 2007;356(2):125-134.

16. Motzer RJ, Hutson TE, Tomczak P, et al. Sunitinib versus interferon alfa in metastatic renal-cell carcinoma. N Engl J Med. 2007;356(2): 115-124.

17. Sternberg CN, Hawkins RE, Wagstaff J, et al. A randomised, doubleblind phase III study of pazopanib in patients with advanced and/or metastatic renal cell carcinoma: final overall survival results and safety update. Eur J Cancer. 2013;49(6):1287-1296.

18. Carmichael C, Lau C, Josephson DY, Pal SK. Comprehensive overview of axitinib development in solid malignancies: focus on metastatic renal cell carcinoma. Clin Adv Hematol Oncol. 2012;10(5):307-314.

19. Hudes G, Carducci M, Tomczak P, et al. Temsirolimus, interferon alfa, or both for advanced renal-cell carcinoma. $N$ Engl J Med. 2007;356(22): 2271-2281.

20. Choueiri TK. Clinical treatment decisions for advanced renal cell cancer. J Natl Compr Canc Netw. 2013;11(5S):694-697.

21. Motzer RJ, Bacik J, Schwartz LH, et al. Prognostic factors for survival in previously treated patients with metastatic renal cell carcinoma J Clin Oncol. 2004;22(3):454-463.
22. Motzer RJ, Escudier B, Bukowski R, et al. Prognostic factors for survival in 1059 patients treated with sunitinib for metastatic renal cell carcinoma. Br J Cancer. 2013;108(12):2470-2477.

23. Heng DYC, Xie W, Regan MM, et al. Prognostic factors for overall survival in patients with metastatic renal cell carcinoma treated with vascular endothelial growth factor-targeted agents: results from a large, multicenter study. J Clin Oncol. 2009;27(34):5794-5799.

24. Motzer RJ, Hutson TE, Tomczak P, et al. Overall survival and updated results for sunitinib compared with interferon alfa in patients with metastatic renal cell carcinoma. J Clin Oncol. 2009;27(22):3584-3590.

25. O'Brien MF, Russo P, Motzer RJ. Sunitinib therapy in renal cell carcinoma. BJU Int. 2008;101(11):1339-1342.

26. Park W-H, Eisen T. Prognostic factors in renal cell cancer. BJU Int. 2007;99(5 Pt B):1277-1281.

27. Schmidinger M, Larkin J, Ravaud A. Experience with sunitinib in the treatment of metastatic renal cell carcinoma. Ther Adv Urol. 2012;4(5): 253-265.

28. George S, Reichardt P, Lechner T, Li S, Cohen DP, Demetri GD. Hypertension as a potential biomarker of efficacy in patients with gastrointestinal stromal tumor treated with sunitinib. Ann Oncol. 2012;23(12):3180-3187.

29. Rini BI, Cohen DP, Lu DR, et al. Hypertension as a biomarker of efficacy in patients with metastatic renal cell carcinoma treated with sunitinib. J Natl Cancer Inst. 2011;103(9):763-773.

30. Rini BI, Tamaskar I, Shaheen P, et al. Hypothyroidism in patients with metastatic renal cell carcinoma treated with sunitinib. $J$ Natl Cancer Inst. 2007;99(1):81-83.

31. Ravaud A, Schmidinger M. Clinical biomarkers of response in advanced renal cell carcinoma. Ann Oncol. 2013;24(12):2935-2942.

32. Wolter P, Stefan C, Decallonne B, et al. Evaluation of thyroid dysfunction as a candidate surrogate marker for efficacy of sunitinib in patients (pts) with advanced renal cell cancer (RCC). J Clin Oncol. 2008:26(Suppl 15):5126.

33. Sabatier R, Eymard JC, Walz J, et al. Could thyroid dysfunction influence outcome in sunitinib-treated metastatic renal cell carcinoma? Ann Oncol. 2012;23(3):714-721.

34. Puzanov I, Michaelson MD, Cohen DP, Li S, Burnett P, Desai J. Evaluation of hand-foot syndrome (HFS) as a potential biomarker of sunitinib (SU) efficacy in patients (pts) with metastatic renal cell carcinoma (mRCC) and gastrointestinal stromal tumor (GIST). Eur J Cancer. 2011;47:Abstr 1444.

35. Donskov F, Michaelson MD, Puzanov I, et al. Comparative assessment of sunitinib-associated adverse events (AEs) as potential biomarkers of efficacy in metastatic renal cell carcinoma (mRCC). Ann Oncol. 2012:23 Suppl 9:Abstr 785O.

36. McDermott DF, Atkins MB. Immune therapy for kidney cancer: a second dawn? Semin Oncol. 2013;40(4):492-498.

37. Yang B, Gu W, Peng B, et al. High level of circulating endothelial progenitor cells positively correlates with serum vascular endothelial growth factor in patients with renal cell carcinoma. J Urol. 2012;188(6): 2055-2061.

38. Vroling L, van der Veldt AA, de Haas RR, et al. Increased numbers of small circulating endothelial cells in renal cell cancer patients treated with sunitinib. Angiogenesis. 2009;12(1):69-79.

39. Rossi E, Fassan M, Aieta M, et al. Dynamic changes of live/apoptotic circulating tumour cells as predictive marker of response to sunitinib in metastatic renal cancer. Br J Cancer. 2012;107(8):1286-1294.

40. Gruenwald V, Beutel G, Schuch-Jantsch S, et al. Circulating endothelial cells are an early predictor in renal cell carcinoma for tumor response to sunitinib. BMC Cancer. 2010;10(1):695.

41. Powles T, Chowdhury S, Shamash J, et al. Increased haematopoietic progenitor cells are associated with poor outcome in patients with metastatic renal cancer treated with sunitinib. Ann Oncol. 2011;22(4):815-820.

42. Choueiri TK, Vaziri SA, Jaeger E, et al. von Hippel-Lindau gene status and response to vascular endothelial growth factor targeted therapy for metastatic clear cell renal cell carcinoma. J Urol. 2008;180(3):860-866. 
43. Kim J, Hwang J, Jeong H, et al. Promoter methylation status of VEGF receptor genes: a possible epigenetic biomarker to anticipate the efficacy of intracellular-acting VEGF-targeted drugs in cancer cells. Epigenetics. 2012;7(2):191-200.

44. Heinrich MC, Maki RG, Corless CL, et al. Primary and secondary kinase genotypes correlate with the biological and clinical activity of sunitinib in imatinib-resistant gastrointestinal stromal tumor. $J$ Clin Oncol. 2008;26(33):5352-5359.

45. Takahashi A, Sasaki H, Kim SJ, et al. Markedly increased amounts of messenger RNAs for vascular endothelial growth factor and placenta growth factor in renal cell carcinoma associated with angiogenesis. Cancer Res. 1994;54(15):4233-4237.

46. Deprimo SE, Bello CL, Smeraglia J, et al. Circulating protein biomarkers of pharmacodynamic activity of sunitinib in patients with metastatic renal cell carcinoma: modulation of VEGF and VEGF-related proteins. J Transl Med. 2007;5:32.

47. Kjeldsen L, Johnsen AH, Sengeløv H, Borregaard N. Isolation and primary structure of NGAL, a novel protein associated with human neutrophil gelatinase. J Biol Chem. 1993;268(14):10425-10432.

48. Porta C, Paglino C, De Amici M, et al. Predictive value of baseline serum vascular endothelial growth factor and neutrophil gelatinase-associated lipocalin in advanced kidney cancer patients receiving sunitinib. Kidney Int. 2010;77(9):809-815.

49. Sakai I, Miyake H, Fujisawa M. Acquired resistance to sunitinib in human renal cell carcinoma cells is mediated by constitutive activation of signal transduction pathways associated with tumour cell proliferation. BJU Int. 2013;112(2):E211-E220.

50. Lieu C, Heymach J, Overman M, Tran H, Kopetz S. Beyond VEGF: inhibition of the fibroblast growth factor pathway and antiangiogenesis. Clin Cancer Res. 2011;17(19):6130-6139.

51. Trikha M, Corringham R, Klein B, Rossi J-F. Targeted anti-interleukin-6 monoclonal antibody therapy for cancer: a review of the rationale and clinical evidence. Clin Cancer Res. 2003;9(13):4653-4665.

52. Nakaigawa N, Yao M, Baba M, et al. Inactivation of von Hippel-Lindau gene induces constitutive phosphorylation of MET protein in clear cell renal carcinoma. Cancer Res. 2006;66(7):3699-3705.
53. Porta C, Paglino C, Imarisio I, et al. Changes in circulating proangiogenic cytokines, other than VEGF, before progression to sunitinib therapy in advanced renal cell carcinoma patients. Oncology. 2013;84(2):115-122.

54. Perez-Gracia JL, Prior C, Guillén-Grima F, et al. Identification of TNFalpha and MMP-9 as potential baseline predictive serum markers of sunitinib activity in patients with renal cell carcinoma using a human cytokine array. Br J Cancer. 2009;101(11):1876-1883.

55. Fujita T, Iwamura M, Ishii D, et al. C-reactive protein as a prognostic marker for advanced renal cell carcinoma treated with sunitinib. Int $J$ Urol. 2012;19(10):908-913.

56. Papazisis KT, Kontovinis LF, Papandreou CN, et al. Brain natriuretic peptide precursor (NT-pro-BNP) levels predict for clinical benefit to sunitinib treatment in patients with metastatic renal cell carcinoma. BMC Cancer. 2010;10:489.

57. Radu A, Pichon C, Camparo P, et al. Expression of follicle-stimulating hormone receptor in tumor blood vessels. N Engl J Med. 2010;363(17): 1621-1630.

58. Siraj MA, Pichon C, Radu A, Ghinea N. Endothelial follicle stimulating hormone receptor in primary kidney cancer correlates with subsequent response to sunitinib. J Cell Mol Med. 2012;16(9):2010-2016.

59. Escudier B, Szczylik C, Porta C, Gore M. Treatment selection in metastatic renal cell carcinoma: expert consensus. Nat Rev Clin Oncol. 2012;9(6):327-337.

60. Porta C, Szczylik C, Escudier B. Combination or sequencing strategies to improve the outcome of metastatic renal cell carcinoma patients: a critical review. Crit Rev Oncol Hematol. 2012;82(3):323-337.

61. Sonpavde G, Choueiri TK, Escudier B, et al. Sequencing of agents for metastatic renal cell carcinoma: can we customize therapy? Eur Urol. 2012;61(2):307-316.
Current Biomarker Findings

\section{Publish your work in this journal}

Current Biomarker Findings is an international, peer-reviewed, open access journal publishing original research, reports, reviews and commentaries on all areas of biomarker research. The manuscript management system is completely online and includes a very quick and fair
Dovepress

peer-review system. Visit http://www.dovepress.com/testimonials.php to read real quotes from published authors. 